\title{
PICOSECOND TRANSIENT ABSORPTION OF XANTHENE DYES*
}

\author{
O. Berndt, F. Bandt, I. Eichwurzel and H. Stiel \\ Max-Born-Institut für Nichlineare Optik und Kurzzeitspektroskopie \\ Rudower Chaussee 6, 12489 Berlin, Germany \\ (Received July 23, 1998; in final form December 28, 1998)
}

After one- and two-colour ps-excitation the transient absorption of rose bengal, erythrosin $\mathrm{B}$ and eosin $\mathrm{Y}$ in the spectral range from 450 to $670 \mathrm{~nm}$ was probed using picosecond continuum pulses generated in $\mathrm{H}_{2} \mathrm{O}-\mathrm{D}_{2} \mathrm{O}$ mixture. Our experiments showed for all dyes a high $T_{1}-T_{n}$ absorption signal above $590 \mathrm{~nm}$ where no ground state absorption occurs. These excited triplet states can be effectively populated by a delayed second pump pulse. Their properties with special attention to reverse intersystem crossing processes from $T_{n}$ to $S_{x}$ are discussed.

PACS numbers: $31.50 .+\mathrm{w}, 33.50 .-\mathrm{j}, 78.47 .+\mathrm{p}$

\section{Introduction}

The excited state photophysical properties of xanthene dyes have both theoretical and practical interest. Xanthene dyes are useful probes for biochemical processes because their photophysical parameters are very sensitive to the environment $[1,2]$. In addition, due to their high efficiency of singlet oxygen generation, xanthene dyes are applied as model systems for photosensitized activity [3]. In laser physics and spectroscopy xanthenes are used as laser dyes or nonlinear materials $[4,5]$.

In some xanthenes a high intersystem crossing rate occurs caused by the internal heavy atom effect [6]. Up to now the properties of the first excited singlet and triplet states $S_{1}, T_{1}$ and the intersystem crossing starting from $S_{1}$ are well known. Reported values of fluorescence quantum yields and lifetimes as well as triplet yields for the xanthene dyes rose bengal, eosin $\mathrm{Y}$ and erythrosin $\mathrm{B}$ are summarized in Table.

Recently for rose bengal, erythrosin $\mathrm{B}$ and eosin $\mathrm{Y}$ a reverse intersystem crossing process from a higher excited triplet state to the singlet system was investigated by one-colour ps-double pulse excitation spectroscopy [7-9]. It was shown

*The results of this paper were initially presented at The Jabtonski Centennial Conference on Luminescence and Photophysics, July 23-27, 1998, Torun', Poland. 
TABLE

Photophysical parameters of xanthene dyes in phosphate buffer.

\begin{tabular}{l|c|c|c}
\hline \hline & Rose bengal & Erythrosin & Eosin Y \\
\hline$\lambda_{\mathrm{abs}}^{\max }[\mathrm{nm}]$ & 549 & 526 & 516 \\
$\sigma_{0}^{\max }\left[10^{-16} \mathrm{~cm}^{2}\right]$ & 3.6 & 3.7 & 3.7 \\
$\lambda_{\mathrm{ffu}}^{\max }[\mathrm{nm}]$ & 569 & 550 & 545 \\
$\phi_{\mathrm{F}}$ & 0.018 & $0.02^{b}$ & $0.20^{b}$ \\
$\tau_{\mathrm{F}}[\mathrm{ps}]$ & 78 & $115^{b}$ & $950^{a}$ \\
$\phi_{\mathrm{ISC}}$ & 0.98 & $0.98^{b}$ & $0.40^{a}$ \\
$k_{\mathrm{ISC}}\left[10^{10} \mathrm{~s}^{-1}\right]$ & 1.0 & $0.85^{b}$ & $0.042^{a}$ \\
\hline \multicolumn{3}{|c}{ data from [8]; data from [6], all other data this work }
\end{tabular}

that this process effectively competes with internal conversion starting from the higher excited triplet state.

An effective population of higher excited triplet and/or singlet states is a prerequisite for photochemical reactions starting from these states. Kochevar et al. [10-12] reported on an oxygen independent photosensitization mechanism in rose bengal starting from a higher excited triplet state. It was shown that a simultaneous two-colour excitation of the dye with ns-pulses at $532 \mathrm{~nm}\left(S_{0}-S_{1}\right.$ absorption band) and $640 \mathrm{~nm}\left(T_{1}-T_{n}\right.$ absorption band) resulted in an enhancement of the photoinhibition of red blood cell acetylcholinesterase as compared with one-colour excitation at $532 \mathrm{~nm}$. The authors concluded that the enhancement of the photosensitizing effect is due to the formation of highly reactive radicals (e.g. the radical anion of the dye) via the higher excited triplet state. The lack of a similar effect for the one-colour excitation regime was interpreted in a model suggesting different deactivation channels of the excited triplet states [12].

The aim of our investigations is to populate definite excited states of xanthenes by stepwise one- or two-colour excitation in order to select specific photophysical or photochemical reaction channels. Therefore at first it is necessary to characterize the photophysical parameters of higher excited states and to understand their kinetics. A useful method is the ps-transient absorption spectroscopy.

\section{Materials and methods}

\subsection{Chemicals}

The xanthenes rose bengal, eosin $Y$ and erythrosin $B$ were supplied by Aldrich (UK). They were used without further purification. The solutions were prepared with bidistilled water using phosphate-buffered saline (PBS) tablets, $\mathrm{pH}=7.4$, from Sigma. All samples were bubbled 30 min with nitrogen to remove the oxygen from the solution. The measurements were carried out in quartz cuvettes (optical path length $1 \mathrm{~cm}$ ) at concentrations $c<10^{-5} \mathrm{M}$. The optical density of the samples at $532 \mathrm{~nm}$ was 0.4 for eosin $\mathrm{Y}$ and 0.6 for erythrosin $\mathrm{B}$ and rose bengal. 


\subsection{Absorption spectra}

Before and after the measurement of a transient absorption spectrum ground state absorption spectra were recorded with an absorption spectrometer (LAMBDA 19, Perkin-Elmer) to determine possible irreversible changes. Ground state absorption spectra of the investigated dyes are represented in Fig. 1.



Fig. 1. Ground state absorption spectra and structure formulae of rose bengal, erythrosin $\mathrm{B}$, and eosin $\mathrm{Y}$.

\subsection{Laser system and continuum generation}

The experimental setup for one- and two-colour transient absorption measurements is shown in Fig. 2. The picosecond pump laser system (502-D.PS 7910, B.M. industries) includes a flashlamp pumped Nd:YAG-laser with active (acousto-optic modulator) and passive (dye cell) mode locking, a double pass amplifier unit, a frequency doubling module (DKDP-crystal, generating the second harmonic "SHG") and the "THG" module (DKDP-crystal), generating the third harmonic by mixing fundamental and SHG. Available wavelengths are $1064 \mathrm{~nm}$ (fundamental), $532 \mathrm{~nm}$ (SHG) and $355 \mathrm{~nm}$ (THG). The pulse energy of the different beams depends on splitting and losses of total energy. Typical ranges of pulse energy and pulse duration are $20-50 \mathrm{~mJ} / 35 \mathrm{ps}$ for fundamental, $8-20 \mathrm{~mJ} / 30 \mathrm{ps}$ for SHG and $8-15 \mathrm{~mJ} / 25 \mathrm{ps}$ for THG at a repetition rate of $10 \mathrm{~Hz}$.

To generate tunable pump pulses a picosecond optical parametric generator (OPG-902, B.M. industries) with two nonlinear anisotropic LBO-crystals pumped 


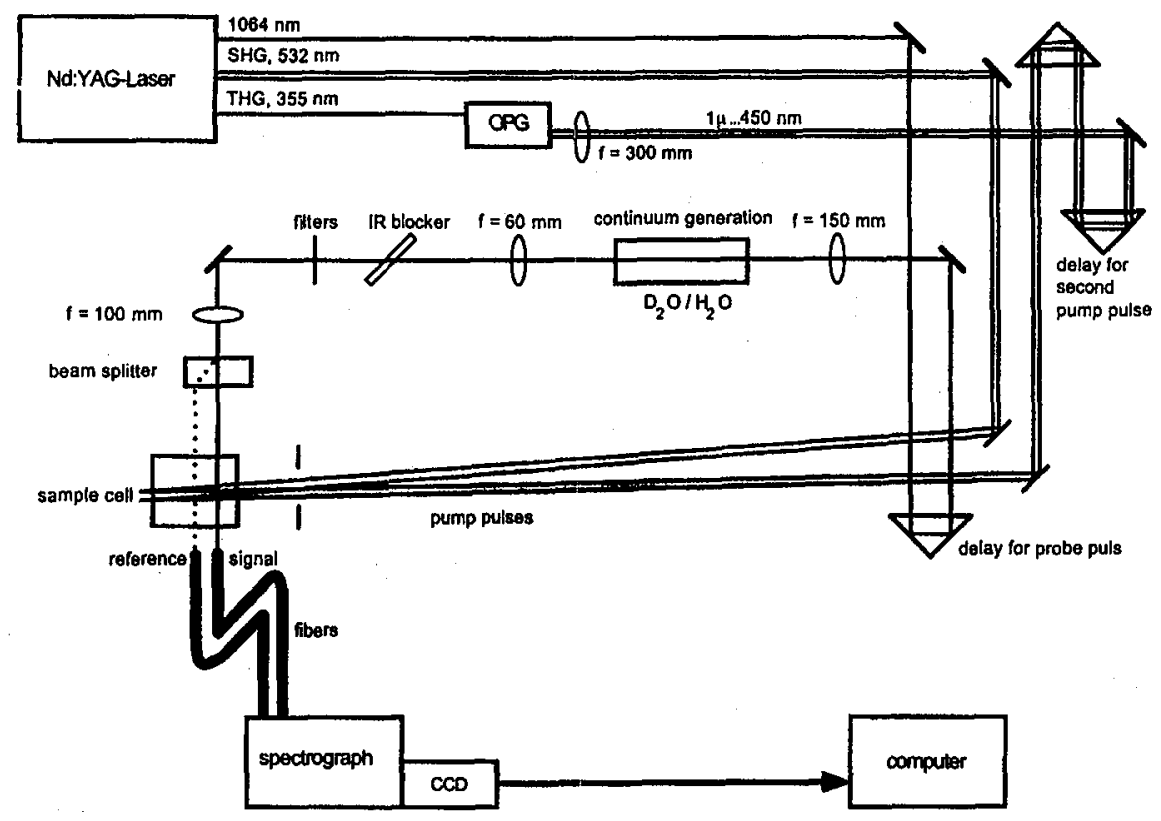

Fig. 2. Pump and probe spectroscopy experimental setup.

by the THG was used. The signal wave was tunable from 440 to $670 \mathrm{~nm}$. The pulse energy at the output was about $100 \mu \mathrm{J}$ and the pulse duration was about 20 ps.

The white light continuum probe pulse (with a pulse duration nearly equal to that of the fundamental) was generated by the fundamental of the Nd:YAG-laser focused into a cuvette (length $10 \mathrm{~cm}$ ) filled with a $\mathrm{H}_{2} \mathrm{O}-\mathrm{D}_{2} \mathrm{O}$ mixture (see Fig. 2). The result of several third order processes, for example stimulated Raman processes and four wave mixing is a broad continuum emission ranging from $440 \mathrm{~nm}$ to far infrared.

\subsection{Optics and detection system}

For time resolved pump-probe measurements variable delay times between pump pulse(s) and probe pulse are necessary. The two delay lines in this experiment for probe pulse and second pump pulse were "optical" lines (moving a prism causes a variation of time delay, see Fig. 2). The delay times could be varied from 0 to 9 ns. The angle between pump beams (which were almost parallel) and probe beam was about $90^{\circ}$ in the sample. Signal and reference beams were carried by fibers from the sample to the detection system. The reflections from front and rear surface of a glass cuboid, positioned in front of the sample and tilted to the beamline were used for separation of signal and reference. For spectral analysis a $0.5 \mathrm{~m}$ Czerny-Turner type spectrograph was connected with a CCD-camera (model 1236, EG \& G Princeton Applied Research). Signal and reference light were detected at the same time and stored by a microprocessor system (IBM-compatible microcomputer model AT 386-25). For each spectrum (and background which 
was subtracted for both CCD signal track and reference track) 200 pulses were accumulated.

\section{Excited state absorption}

\subsection{Theory}

For most transient absorption experiments a one-dimensional model can be applied. The spectrum of transmitted light can be described by the spectral shape function

$$
S \propto \mathrm{d} I / \mathrm{d} x \propto-\langle\boldsymbol{E} \cdot(\partial \boldsymbol{P} / \partial t)\rangle \propto-N \sum_{i}\left(n_{i} \sum_{k} \sigma_{i \rightarrow k}\right) I,
$$

where $S=$ spectral shape function, $I=$ intensity, $\boldsymbol{E}=$ electric field vector, $\boldsymbol{P}=$ polarization vector, $N=$ density of absorbing particles, $n_{i}=$ relative population for state $i\left(\sum_{i} n_{i}=1\right)$ and $\sigma_{i \rightarrow k}=$ cross-section for transition $i \rightarrow k$. The optical density $O D$ of a sample with optical path length $d$ is given by

$O D(\lambda, t)=(1 / \ln 10) N \int_{0}^{d}\left[\sum_{i=0} \sum_{k>i} n_{i}(t, x) \sigma_{i \rightarrow k}-\sum_{i=1} \sum_{i<k} n_{k}(t, x) \sigma_{k \rightarrow i}\right] \mathrm{d} x$,

where $E_{i}<E_{k}$ for $i<k$ (energy levels), $n_{i}=\sum_{v, r} n_{i, v, r}(v, r$ are indices of vibronic sublevels), $\sigma_{n \rightarrow m}=f(\lambda)$. The first term on the right side in Eq. (2) describes the absorption processes and the second term describes the emission processes. In transient absorption spectroscopy the difference of optical densities $\triangle O D$ with and without excitation (pump pulse) is measured

$$
\Delta O D(\lambda, \Delta t)=O D_{\mathrm{ex}}(\lambda, \Delta t)-O D_{\mathrm{gs}}(\lambda, \Delta t)=-\log \left[T_{\mathrm{ex}}(\lambda, t) / T_{\mathrm{gs}}(\lambda)\right],
$$

where $O D_{\mathrm{ex}}(\lambda, \Delta t), T_{\mathrm{ex}}(\lambda, t)=$ optical density and transmission for excited sample and $O D_{\mathrm{gs}}(\lambda, \Delta t), T_{\mathrm{gs}}(\lambda)=$ optical density and transmission for non-excited sample.

For long delay times $\Delta t$ between pump and test pulse only contributions starting from long-lived states, for example triplet states and bleaching (depletion of ground state) are expected. For short delay times a mixture of contributions (bleaching, absorption, and induced emission) of fast processes is expected. The contribution of induced emission can be described by the fluorescence spectrum because of the connection of Einstein's $A$ and $B$ coefficients assuming a very short Franck-Condon relaxation time of vibronic states of $S_{1}$ compared to pulse duration. The result for the induced emission cross-section [13] is

$$
\sigma_{\text {ind }}(\lambda)=\frac{\lambda^{4}}{8 \pi c_{0} n^{2}} \frac{\phi_{\mathrm{F}}}{\tau_{\mathrm{F}}} g(\lambda)
$$

where $c_{0}=$ vacuum light velocity, $n=$ refractive index of the sample, $\phi_{\mathrm{F}}=$ fluorescence quantum yield, $\tau_{\mathrm{F}}=$ fluorescence lifetime and $g(\lambda)=$ shape function of fluorescence, where $\int_{0}^{\infty} g(\lambda) \mathrm{d} \lambda=1$.

For one-colour measurements difference spectra are given by the expression (3) while for two-colour excitation the related expression is $\triangle O D^{\prime}=$ $\log \left(T_{\mathrm{gs}} / T_{\mathrm{ex}}^{\prime}\right)$, where $T_{\mathrm{ex}}^{\prime}=$ transmission of sample, excited by both of the pump pulses. The variation in spectra caused by addition of the second pump field can be determined by the expression

$$
\Delta(\triangle O D)=\triangle O D^{\prime}-\triangle O D=\log \left(T_{\mathrm{ex}} / T_{\mathrm{ex}}^{\prime}\right)
$$


The fundamental equations for theoretical description of incoherent interaction of matter and light are the photon transport equation and the rate equations. The photon transport equation for a pump pulse propagating in $x$-direction is given by

$$
\left(\frac{1}{c} \frac{\partial}{\partial t}+\frac{\partial}{\partial x}\right) I(x, y, z, t)=-I(x, y, z, t) N \sum_{i} \sum_{j} \sigma_{i j}\left(n_{i}-n_{j}\right) .
$$

The rate equations are given by

$$
\frac{\mathrm{d} n_{i}}{\mathrm{~d} t}=\sum_{j} \sigma_{i j}\left(n_{j}-n_{i}\right) I+\sum_{j>i} k_{j i} n_{j}-\sum_{j<i} k_{i j} n_{i},
$$

where $k_{i j}=$ rate constant of relaxation process from state $j$ to state $i$.

\subsection{Calibration of zero delay time point}

For time resolved measurements a time calibration is necessary. A simple method to find maximum overlap of pump and probe pulse is to make use of bleaching effects in rose bengal because of its short-lived $S_{1}$-state and high intersystem crossing (ISC) quantum yield (see Table). For first approximation the bleaching effects in rose bengal can be described by a three-level model containing only $S_{0^{-}}, S_{1^{-}}$and $T_{1}$-state [14]. The $T_{1}$-state acts as a reservoir. Because of a negligible small $S_{1} \rightarrow S_{x}$ absorption cross-section at $532 \mathrm{~nm}$ [14] only the first equation of the following simple rate equation system has to be considered:

$$
\begin{aligned}
\frac{\mathrm{d} n_{0}}{\mathrm{~d} t} & =-\sigma_{0} I n_{0}+\left(k_{\mathrm{R}}+k_{\mathrm{IC}}\right) n_{1}, \\
\frac{\mathrm{d} n_{1}}{\mathrm{~d} t} & =\sigma_{0} I n_{0}+\left(k_{\mathrm{R}}+k_{\mathrm{IC}}+k_{\mathrm{ISC}}\right) n_{1}, \\
\frac{\mathrm{d} n_{\mathrm{T}}}{\mathrm{d} t} & =k_{\mathrm{ISC}} n_{1}=-\frac{\mathrm{d}}{\mathrm{d} t}\left(n_{0}+n_{1}\right),
\end{aligned}
$$

where $n_{0}=n\left[S_{0}\right], n_{1}=n\left[S_{1}\right], n_{\mathrm{T}}=n\left[T_{1}\right] ; \sigma_{0}=$ ground state absorption cross-section, $k_{\mathrm{R}}, k_{\mathrm{IC}}$ and $k_{\mathrm{ISC}}=$ rate constants of radiation, internal conversion and intersystem crossing.

Neglecting the last term in the first equation of (8) it can be integrated separately

$$
n_{0}(t)=n_{0}(t \rightarrow-\infty) \exp \left(-\sigma_{0} \int_{-\infty}^{t} I\left(t^{\prime}\right) \mathrm{d} t^{\prime}\right)
$$

with $n_{0}(t \rightarrow-\infty)=1$.

The exponential function can be evolved and for small intensities the expression becomes

$$
n_{0}(t)=1-\sigma_{0} \int_{-\infty}^{t} I\left(t^{\prime}\right) \mathrm{d} t^{\prime}
$$

For a rough estimation we assume a narrow probe beam propagating perpendicular to pump beam in $x$-direction ( $x=0$ at half of the length $d$ of the sample cuvette) characterized by

$$
I(x, t)=I_{0}(x) \frac{1}{\left(t-t_{0}\right)^{2} / \tau_{\mathrm{L}}^{2}+1}
$$


where $\tau_{\mathrm{L}}=$ pump pulse duration, $t_{0}=-x / c_{n}, c_{n}=c_{0} / n=$ light velocity in the sample and $I_{0}(x)=$ transversal shape of pump pulse, $I_{0}(x)=I_{0}(-x)$. Equation (10) now becomes

$$
n_{0}(t, x)=1-\tau_{\mathrm{L}} \sigma_{0} I_{0}(x)\left[\arctan \left(\frac{t+x / c_{n}}{\tau_{\mathrm{L}}}\right)+\pi / 2\right] .
$$

The contribution of higher excited state absorption in the maximum of bleaching spectrum can be neglected (see above). The variation of optical density along the path of the probe pulse is given by

$$
\mathrm{d}(O D(t, x))=N\left(\sigma_{0} / \ln 10\right) n_{0}(t, x) \mathrm{d} x .
$$

For finite duration of probe pulse $\tau_{\mathrm{P}}$ and $I_{\mathrm{P}}(t) \propto S_{\mathrm{P}}(t)=S_{\mathrm{P}}(-t)$ (where $\left.\int_{-\infty}^{\infty} S_{\mathrm{P}}(t) \mathrm{d} t=1\right)$ the total optical density is a convolution of the expression on the right side of (13) with the shape function $S_{\mathrm{P}}$ integrated over the path of the probe light

$$
O D(t)=c_{1}-c_{2} \int_{-t^{\prime \prime 2}}^{d / 2} I_{0}(x) \int_{-\infty}^{\infty} S_{P}\left(t^{\prime}\right) \arctan \left(\frac{t-t^{\prime}+x / c_{n}}{\tau_{\mathrm{L}}}\right) \mathrm{d} t^{\prime} \mathrm{d} x
$$

where $c_{1}$ and $c_{2}=$ constants $>0$. For $t \rightarrow-\infty$ Eq. (14) yields

$$
O D(t)=c_{1}+c_{2} \int_{-d / 2}^{d / 2} I_{0}(x) \frac{\pi}{2} \mathrm{~d} x=c_{1}+c_{3},
$$

where $c_{3}=$ constant $>0$. For $t \rightarrow \infty$ one obtains $O D(t)=c_{1}-c_{3}$, and for $t=0$ Eq. (14) becomes $O D(t)=c_{1}$ because of symmetry of $I_{0}(x)$ and antisymmetry of

$$
\int_{-\infty}^{\infty} S_{\mathrm{P}}\left(t^{\prime}\right) \arctan (t=0, x, \ldots) \mathrm{d} t^{\prime}
$$

in $x$. It is easy to see that for $t=0$ (maximum of pulse overlap) bleaching takes half of its maximal value. Varying the delay of probe pulse one can find the time zero point looking for half of the maximum bleaching. Applying this method one has to use pump intensities definitely below saturation of the bleaching because otherwise half of maximum bleaching value will be exceeded before $t=0$.

The described procedure was applied in our experiments for determination of zero delay time point. For the measurement of transient absorption spectra higher intensities were applied and saturation effects occurred (see next section).

\section{Experimental results}

\subsection{Transient spectra and kinetics}

In this section the transient spectra of rose bengal, erythrosin $\mathrm{B}$ and eosin $\mathrm{Y}$ are represented. The SHG $(\lambda=532 \mathrm{~nm}$, pulse energy $=1.2 \mathrm{~mJ}$, beam diameter in the sample $=4 \mathrm{~mm}$, assuming a Gaussian beam; $I=10^{27} \mathrm{~cm}^{-2} \mathrm{~s}^{-1}$ ) was used for one-colour excitation. The path length of the probe beam in the sample was $1 \mathrm{~cm}$. The delay times between pump and probe pulse ranged from $-75 \mathrm{ps}$ to $8.5 \mathrm{~ns}$.

In Figs. 3a-5a transient absorption spectra of rose bengal, erythrosin B and eosin $\mathrm{Y}$ are shown measured at different time delays after excitation at the $S_{0}-S_{1}$ absorption band. For all investigations at a zero time delay a strong bleaching 

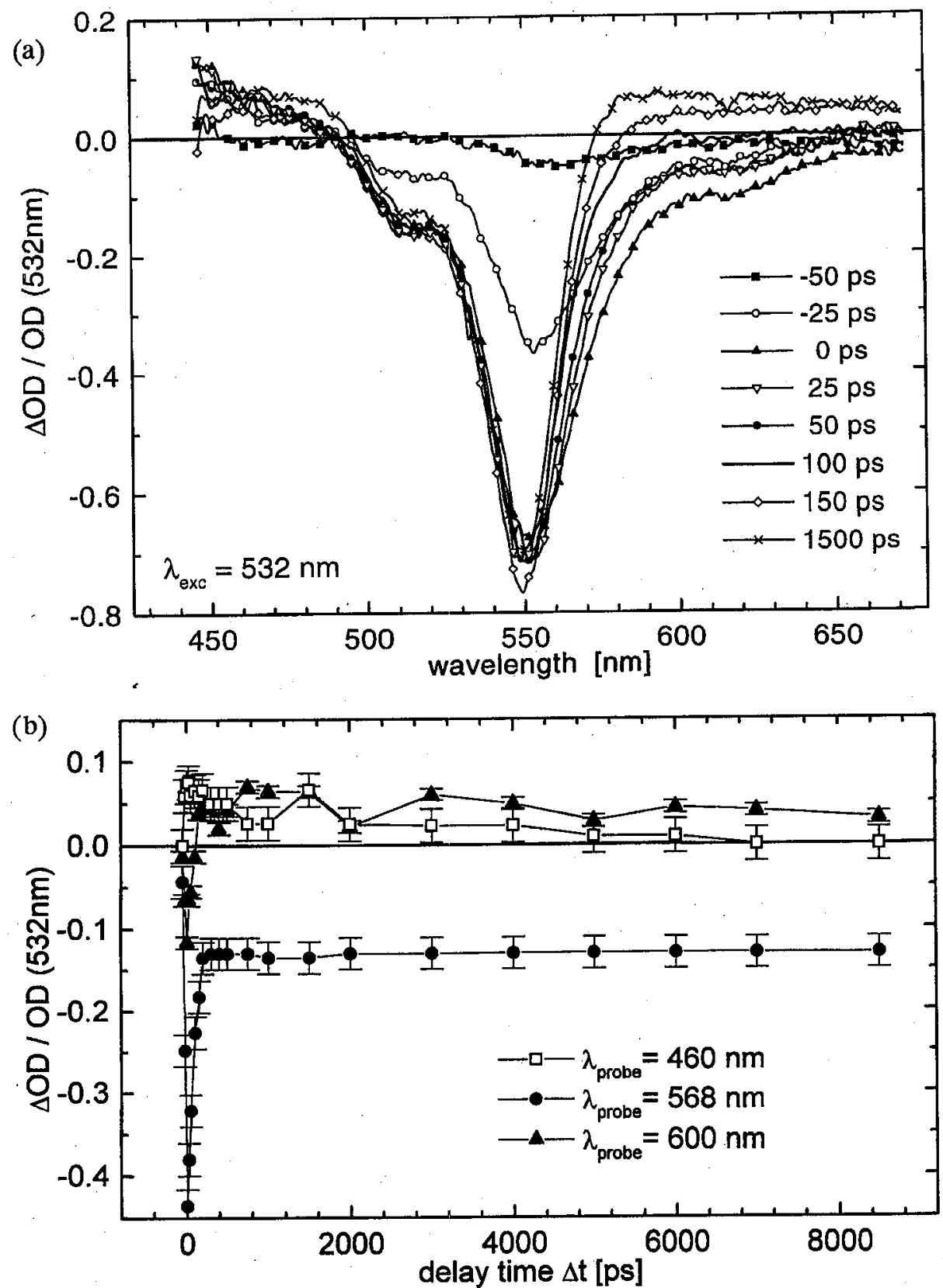

Fig. 3. (a) Transient absorption spectra of nitrogen bubbled rose bengal at different delay times between pump pulse $\left(\lambda_{1}=532 \mathrm{~nm}\right)$ and probe pulse; (b) dependence of $\triangle O D$ (rose bengal) on time delay between pump pulse $\left(\lambda_{1}=532 \mathrm{~nm}\right)$ and probe pulse at three different probe wavelengths. 

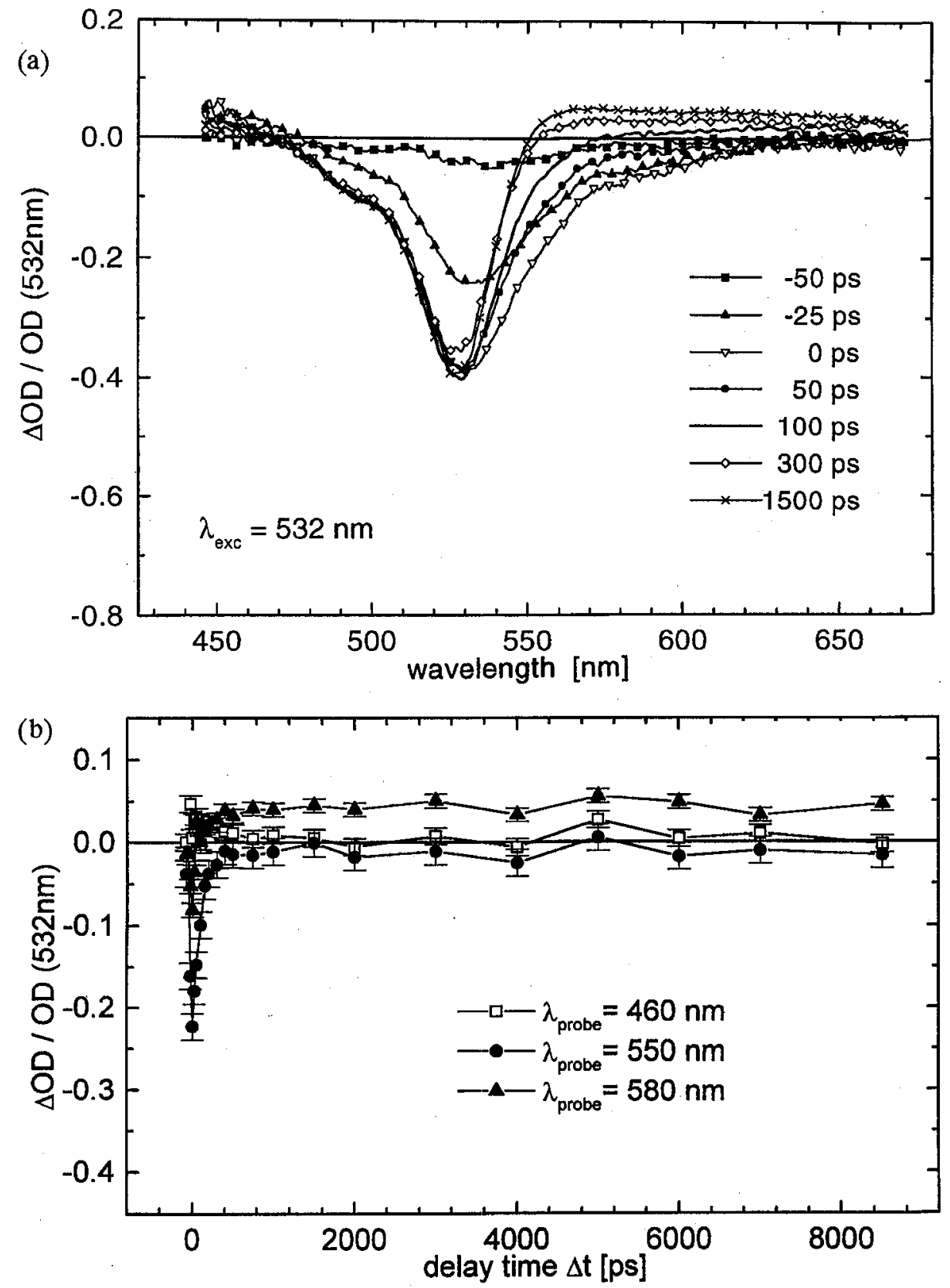

Fig. 4. (a) Transient absorption spectra of nitrogen bubbled erythrosin $B$ at different delay times between pump pulse $\left(\lambda_{1}=532 \mathrm{~nm}\right)$ and probe pulse; (b) dependence of $\triangle O D$ (erythrosin B) on time delay between pump pulse $\left(\lambda_{1}=532 \mathrm{~nm}\right)$ and probe pulse at three different probe wavelengths. 



Fig. 5. (a) Transient absorption spectra of nitrogen bubbled eosin $Y$ at different delay times between pump pulse $\left(\lambda_{1}=532 \mathrm{~nm}\right)$ and probe pulse; (b) dependence of $\triangle O D$ (eosin $Y$ ) on time delay between pump pulse $\left(\lambda_{1}=532 \mathrm{~nm}\right)$ and probe pulse at three different probe wavelengths. 
around the $S_{0}-S_{1}$ transition occurs. This decrease in $O D$ is due to a superposition of at least two processes: saturation of the $S_{0}-S_{1}$ absorption and induced emission from the $S_{1}$ state. The latter process is characterized by the emission cross-section $\sigma_{\text {ind }}$ (cp. Eq. (4)). Its maximum value is Stokes shifted with respect to the absorption maximum of the dyes (cp. Table). The contribution of both processes leads to a shift of the bleaching maximum to longer wavelengths with respect to the ground state absorption maximum. In particular if the fluorescence lifetime is much longer than the pulse duration as in the case of eosin $\left(\tau_{\mathrm{F}}=950 \mathrm{ps}\right.$, $\mathrm{cp}$. Table) the bleaching maximum shifts to shorter wavelengths at longer delay times (Fig. 5a).

As can be seen from Figs. 3a-5a for all three dyes an excitation induced broad transient absorption band occurs between 580 and $650 \mathrm{~nm}$ with a maximum $\triangle O D$-value between 0.05 (erythrosin $\mathrm{B}$ ) and 0.07 (rose bengal). At long delay times this value remains nearly unchanged. In correspondence with Refs. $[9,12]$ we assign this absorption to the triplet-triplet absorption. The relatively fast occurrence of the triplet-triplet absorption (cp. Figs. 3b-5b) indicates an effective intersystem crossing process for rose bengal and erythrosin $\mathrm{B}$.

An additional absorption band was found for all dyes at shorter wavelengths around $460 \mathrm{~nm}$. This band having a maximum $\triangle O D$-value between 0.03 (erythrosin $B$ ) and 0.07 (rose bengal) rapidly vanished simultaneously with the stimulated emission at longer delay times (cp. Figs. 3b-5b). Due to its short lifetime we interpret this absorption band as the singlet-singlet absorption.

\subsection{Two-colour excitation}

In order to prove both radical formation via higher excited triplet state as described by Kochevar et al. [11] and back intersystem crossing [7] from an excited triplet state $T_{n}$ to $S_{x}$ (cp. Fig. 6) two-colour excitation measurements were performed.

In these experiments the sample was excited by both the SHG of the $\mathrm{Nd}: Y A G-l a s e r$ at $\lambda_{\text {exc }}=532 \mathrm{~nm}$ and the signal wave of the OPG tuned to the maximum of the $T_{1}-T_{n}$ absorption band $\left(\lambda_{2}=600-640 \mathrm{~nm}\right)$. The delay time between the two pump pulses was set to $1 \mathrm{~ns}$.

The other parameters were: $E_{1}=1.2 \mathrm{~mJ}, I_{1}=10^{27} \mathrm{~cm}^{-2} \mathrm{~s}^{-1}$, beam diameter $4 \mathrm{~mm}, E_{2}(600 \mathrm{~nm})=30 \mu \mathrm{J}, I_{2}(600 \mathrm{~nm})=1.7 \times 10^{26} \mathrm{~cm}^{-2} \mathrm{~s}^{-1}$, elliptical beam $3 \mathrm{~mm} \times 1 \mathrm{~mm}$.

The probe pulse delay was varied between $-50 \mathrm{ps}$ and $4 \mathrm{~ns}$ relative to the OPG pulse.

Transient absorption spectra of rose bengal were recorded for different delay times between the probe pulse and the excitation pulses. Only spectra recorded at delay times between $-25 \mathrm{ps}$ and $+100 \mathrm{ps}$ showed an additional increase in the $\triangle O D$ (cp. Fig. 7). At longer delay times no changes of $\triangle O D$ were detected (data not shown). The induced increase in $\triangle O D$ is located in the wavelength region around the $S_{0}-S_{1}$ transition at $550 \mathrm{~nm}$. Therefore, we assign the appearance of this band to a fast intersystem crossing process $T_{n} \rightarrow S_{x}$ leading to a re-population of the excited singlet state $S_{1}$. 


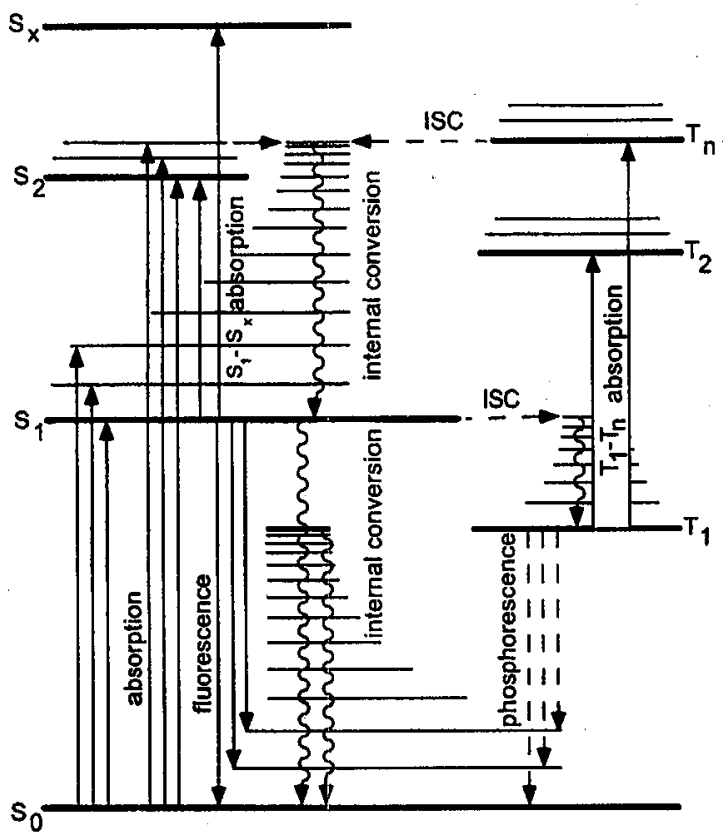

Fig. 6. Jabłoński diagram of energy levels for xanthene dyes.

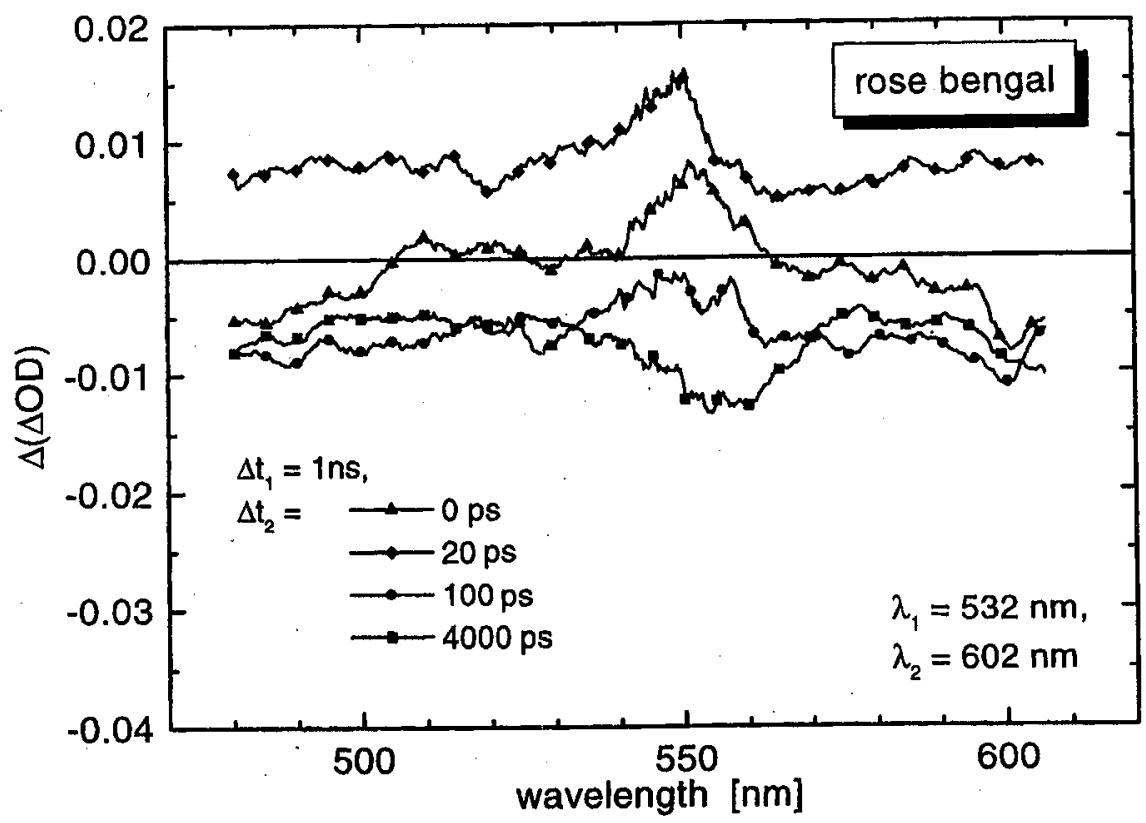

Fig. 7. Transient two-colour absorption spectra $\Delta(\triangle O D)$ of rose bengal at 1 ns delay time between first pump pulse $\left(\lambda_{1}=532 \mathrm{~nm}\right)$ and second pump pulse $\left(\lambda_{2}=602 \mathrm{~nm}\right)$. 


\section{Conclusions}

Using one- and two-colour ps-excitation we have studied transient absorption processes in the xanthenes rose bengal, erythrosin B and eosin Y. At short delay times we have found for all dyes an absorption band located at $460 \mathrm{~nm}$. Due to its short lifetime this band was assigned to excited singlet state absorption.

At longer delay times a transient absorption at wavelengths above $570 \mathrm{~nm}$ occurs. In correspondence with data in $[7,16]$ this process can be explained by triplet-triplet absorption. The excited singlet and triplet state absorption cross-sections can be calculated from the measured transient absorption spectra according to a procedure described in $[14,15]$.

The transient absorption measurements after two-colour excitation of rose bengal yielded an indication of intersystem crossing process from the higher excited triplet state $T_{n}$ to the excited singlet state $S_{x}$ followed by a fast internal conversion to the $S_{1}$-state. We did not find any indication for a transient absorption of photoproducts in the time range between 0 and $4 \mathrm{~ns}$ after two-colour excitation of rose bengal. The generation of photoproducts such as the semi-oxidized radical with absorption maxima around $470 \mathrm{~nm}$ via higher excited triplet state was proposed by Kochevar et al. [11] as a prerequisite for a new, oxygen independent sensitization mechanism in rose bengal.

\section{Acknowledgment}

We acknowledge D. Leupold from MBI for valuable discussions.

\section{References}

[1] J.C. del Valle, J. Catalan, F. Amat-Guerri, J. Photochem. Photobiol. A, Chem. 72, 49 (1993).

[2] D.C. Neckers, J. Photochem. Photobiol. A, Chem. 47, 1 (1989).

[3] E.N. Fluhler, J.K. Hurley, I.E. Kochevar, Biochem. Biophys. Acta 990, 269 (1989).

[4] A.V. Deshpande, N.B. Iyer, Chem. Phys. Lett. 157, 239 (1989).

[5] S. Speiser, D. Dantsker, J. Appl. Phys. 66,61 (1989).

[6] G.R. Fleming, A.W.E. Knight, J.M. Morris, R.J.S. Morrison, G.W. Robinson, JACS 99, 4306 (1977).

[7] S. Reindl, A. Penzkofer, Chem. Phys. 211, 431 (1996).

[8] S. Reindl, A. Penzkofer, Chem. Phys. 213, 429 (1996).

[9] A. Penzkofer, A. Beidoun, Chem. Phys. 177, 203 (1993).

[10] M.T. Allen, M. Lynch, A. Lagos, R.W. Redmond, I.E. Kochevar, Biochem. Biophys. Acta 1075, 42 (1991).

[11] G. Smith, W.G. McGimpsey, M.C. Lynch, I.E. Kochevar, R.W. Redmond, Photochem. Photobiol. 59, 135 (1994).

[12] C.R. Lambert, H. Stiel, D. Leupold, M.C. Lynch, I.E. Kochevar, Photochem. Photobiol. 63, 154 (1996).

[13] O.G. Peterson, J.P. Webb, W.C. McColgin, J.H. Eberly, J. Appl. Phys. 42, 1917 (1971). 
[14] H. Stiel, K. Teuchner, A. Paul, D. Leupold, I.E. Kochevar, J. Photochem. Photobiol. B, Biology 33, 245 (1996).

[15] H. Stiel, K. Teuchner, J. Ehlert, S. Oberlaender, D. Leupold, Intell. Instr. Comp. 9, 79 (1992).

[16] F. Bandt, Master's thesis, Humboldt-Universität zu Berlin, 1998. 